\section{The Role of Nuclear Medicine for COVID-19: Time to Act Now}

TO THE EDITOR: As every medical department worldwide is bracing for the impact the coronavirus disease 2019 (COVID-19) pandemic will pose on daily routines, the scientific community starts to excel in exploring the different facets this novel disease bears for prevention, diagnosis, and treatment. Nuclear medicine, touting itself "molecular medicine" as well as "theranostic medicine" may not fall short here, as we have much to offer.

Although it may be wise for individual nuclear medicine departments to focus on taking precautions for themselves and their most vulnerable patients, with oncologic and cardiovascular or neurologic "predispositions," to the risks of exposure to COVID19-bearing individuals (1), we should still motivate ourselves and the academic nuclear medicine community to actively participate in improving care for COVID-19 patients.

Accumulating evidence suggests that some of the detrimental effects seen in patients with severe COVID-19 are attributed to an overly host antiviral defense as seen in severe acute respiratory syndrome (SARS), leading to hyperinflammatory reactions or cytokine storm syndrome, sometimes also affecting the central nervous system (CNS) (2,3).

Until now, however, existing knowledge regarding supportive care and adjunctive pharmacologic therapy is limited. Even worse, a subgroup of COVID-19 patients that seems to do well after getting out of the intensive care unit dies of acute respiratory syndrome just several days later, without clinical signs indicating their imminent deterioration. This situation may be one of the first places where nuclear medicine should tune in: With ${ }^{18} \mathrm{~F}-\mathrm{FDG}$ PET/CT for decades being well evaluated for its sensitivity in detecting inflammatory disease (4), we should start to prospectively collect data in a well-defined group of patients at given time intervals during the course of COVID-19 infection to better understand the inflammatory component of the disease and maybe find early prognostic signs that warrant proactive antiinflammatory treatment in patients at risk. Until now, only anecdotal data exist (5). As a nuclear medicine community, we ought to team up and establish protocols suitable for multicentric evaluation as soon as possible, meeting the requirements for controlled trials. Serial examinations should include both cohorts of patients with proven COVID-19 with different severity and extended follow-up after recovery as well as symptomatic patients with radiographic findings typical for COVID-19, but without initial proof of infection, and should not only focus on pulmonary inflammation but also address possible inflammatory involvement (e.g., of myocardium, pericardium, vasculature, muscles, intestine, and the CNS.

At academic sites providing research facilities including cyclotron and radiopharmacy production, the research into the inflammatory cascade could go even further. There are well-established radiopharmaceuticals suitable as inflammatory biomarkers at an intracellular level, for example, targeting the purinergic P2X7 receptor (6). Although initially designed to quantify neuroinflammation, they can easily be repurposed for imaging the inflamma-

Immediate Open Access: Creative Commons Attribution 4.0 International License (CC BY) allows users to share and adapt with attribution, excluding materials credited to previous publications. License: https://creativecommons. org/licenses/by/4.0/. Details: http://jnm.snmjournals.org/site/misc/permission. xhtml. some and quantifying inflammation at a whole-body level. As there are also potential $\mathrm{P} 2 \mathrm{X} 7$ inhibitors at the receptor level (6), showing antiinflammatory effects in animal models (7), translational research may here form the rationale for antiinflammatory therapy principles in COVID-19. Furthermore, nuclear medicine has the potential to provide evidence and clarify contradictory concepts in the use of nonsteroidal antiinflammatory drugs in COVID-19, where clinicians have to state, "many clinical anecdotes remain stalled in biologic plausibility" (8), by directly depicting cyclooxygenase-2 (COX2) involvement using established COX2inhibitory radiopharmaceuticals (9).

Possible repurposing of other established radiopharmaceuticals to investigate COVID-19-specific pathomechanisms might target the cytokine signaling pathway (e.g., chemokine receptor CXCR4, interleukin IL-6), fibroblast activation protein inhibitors (FAPI), to address postinflammatory fibrosis, or inhibitors of the type 1 angiotensin-II-receptor ATR1 (e.g., KR31173), involved in cellular internalization of SARS-CoV-2 (2). Development of novel radiopharmaceuticals could also focus on directly targeting the entry receptor for SARS-CoV-2, the angiotensin-converting-enzyme-2 (ACE2). Radiolabeling of an ACE2-receptor antagonist has already been achieved for receptor autoradiography protocols (10) and could serve as a starting point for PET tracer development, increasing our readiness for the next coronavirus shift.

The recent World Health Organization initiative of creating a voluntary intellectual property pool for COVID-19 products to balance intellectual property and accessibility could address some of the issues inhibiting broader application of new tracers. Regulatory agencies also recently have shown extraordinary performance in overviewing new applications. And nuclear medicine already has proven in many ways to excel in logistics for radiopharmaceutical distribution, partnering with academic, administration, and industrial stakeholders.

The examples chosen here are not meant to be comprehensive. They are meant to stimulate our community's potential to contribute to one of the biggest challenges in modern medicine. The existing Society of Nuclear Medicine and Molecular Imaging Connect platform may serve as a natural vehicle to collectively define and distribute protocols suitable for multicentric trials and to share what it needs for making novel radiopharmaceuticals accessible at an accelerated time scale.

Let us build a network of clinical trials, let us start now and fast and bold. It is time to act now.

\section{DISCLOSURE}

No potential conflict of interest relevant to this article was reported.

\section{REFERENCES}

1. Czernin J, Fanti S, Meyer PT, et al. Nuclear medicine operations in the times of COVID-19: strategies, precautions, and experiences. J Nucl Med. April 1, 2020 [Epub ahead of print].

2. Fung S-Y, Yuen K-S, Ye Z-W, Chan C-P, Jin D-Y. A tug-of-war between severe acute respiratory syndrome coronavirus 2 and host antiviral defence: lessons from other pathogenic viruses. Emerg Microbes Infect. 2020;9:558-570.

3. Mehta P, McAuley DF, Brown M, et al. COVID-19: consider cytokine storm syndromes and immunosuppression. Lancet. 2020;395:1033-1034.

4. Treglia G. Diagnostic performance of ${ }^{18} \mathrm{~F}-\mathrm{FDG}$ PET/CT in infectious and inflammatory diseases according to published meta-analyses. Contrast Media Mol Imaging. 2019;2019:3018349.

5. Qin C, Liu F, Yen T-C, Lan X. ${ }^{18}$ F-FDG PET/CT findings of COVID-19: a series of four highly suspected cases. Eur J Nucl Med Mol Imaging. 2020;47:1281-1286.

6. Koole M, Schmidt ME, Hijzen A, et al. ${ }^{18}$ F-JNJ-64413739, a novel PET ligand for the P2X7 ion channel: radiation dosimetry, kinetic modeling, test-retest variability, 
and occupancy of the P2X7 antagonist JNJ-54175446. J Nucl Med. 2019;60:683690.

7. Duan L, Hu G, Li Y, Zhang C, Jiang M. P2X7 receptor is involved in lung injuries induced by ischemia-reperfusion in pulmonary arterial hypertension rats. Mol Immunol. 2018;101:409-418.

8. FitzGerald GA. Misguided drug advice for COVID-19. Science. 2020;367:1434

9. Bhardwaj A, Kaur J, Wuest M, Wuest F. In situ click chemistry generation of cyclooxygenase-2 inhibitors. Nat Commun. 2017;8:1.

10. Linares A, Couling LE, Carrera EJ, Speth RC. Receptor autoradiography protocol for the localized visualization of angiotensin II receptors. J Vis Exp. 2016;112:1-15.

Freimut D. Juengling* Antonio Maldonado Frank Wuest

Thomas Hellmut Schindler *University Bern Murtenstrasse 11 Bern, Switzerland 3008 E-mail: freimut.juengling@med.unibe.ch

Published online Apr. 17, 2020.

DOI: 10.2967/jnumed.120.246611

\section{Who Was the First Doctor to Report the COVID-19 Outbreak in Wuhan, China?}

TO THE EDITOR: We read with great interest the recent publication entitled "Dr. Li Wenliang and the Time of COVID-19" (1). As mentioned in this article, Dr. Li was reprimanded initially for "disrupting public order" in China when he first reported the outbreak in Wuhan. As a matter of fact, On December 30, 2019, Dr. Li warned in an online chat group on WeChat that he had seen a report showing positive test results of SARS for 7 patients. However, he did not formally report the outbreak to the authorities.

Dr. Zhang Jixian is considered the first doctor to report the novel coronavirus before its outbreak. A senior couple living in the residential community near Dr. Zhang's hospital went to see her for their fever and cough on December 26, 2019. When she observed their CT thorax images, Dr. Zhang found differences from pneumonia caused by common viruses. Zhang's experience during the 2003 SARS outbreak, when she worked as a medical expert investigating suspected patients in Wuhan, made her sensitive to signs of an epidemic. After reading the CT images of the elderly couple, she summoned their son, demanding a CT scan of him too. It was Zhang's insistence that brought her the second piece of evidence: the son's lungs showed the same abnormalities as those of his parents. Also on December 27 , the hospital received another patient who also developed symptoms of coughing and fever and showed the same lung images in the CT scan. The blood tests of the 4 indicated viral infections.

"Usually, a family comes to the hospital and there is little chance for all the family members to have the same disease except

Immediate Open Access: Creative Commons Attribution 4.0 International License (CC BY) allows users to share and adapt with attribution, excluding materials credited to previous publications. License: https://creativecommons. org/licenses/by/4.0/. Details: http://jnm.snmjournals.org/site/misc/permission. xhtml. for infectious diseases," said Dr. Zhang, who gave the couple's family and the patient from the seafood market tests denying the possibility of flu.

As the situation continued to confuse Zhang, she reported it to the head of the hospital on December 27, and the hospital then reported it to the Center for Disease Control (CDC) in the Jianghan district of Wuhan. Back then, knowledge of the virus was scarce. After filing the report, Dr. Zhang cordoned off an area in the department's ward to hospitalize the 4 patients described above. She then demanded medics in the ward to beef up selfprotection. Epidemiologic investigations and tests were arranged quickly by the CDC. Within $2 \mathrm{~d}$, the hospital continued to receive more similar patients, and Dr. Zhang promptly reported to the hospital. Before the CDC expert group paid attention to this epidemic, Dr. Zhang had set up a 9-bed isolation ward for the patients and taken measures to isolate them. She also bought 30 pieces of canvas as protective clothing. "If it is delivered uniformly by the hospital, it needs to be customized" she said, "if it is online shopping, they can receive the goods very quickly."

At 1:00 PM on December 29, the vice president of the hospital reported directly to the disease control department of the provincial and municipal health and Health Commission. Although December 29 is not a working day, the provincial and municipal health commission disease control department immediately responded to the report and came to the hospital to formally begin the epidemiologic investigation.

At 3:10 PM on December 30, Wuhan Municipal Health and Health Commission issued the official document "emergency notice on reporting the treatment of pneumonia of unknown causes." The National Health Commission (NHC) dispatched a working group and an expert team in the wee hours of December 31 to Wuhan to guide epidemic response and conduct on-site investigations. Not only was Dr. Zhang the first to sound an alarm for epidemic prevention and control, but she was also the "leader"

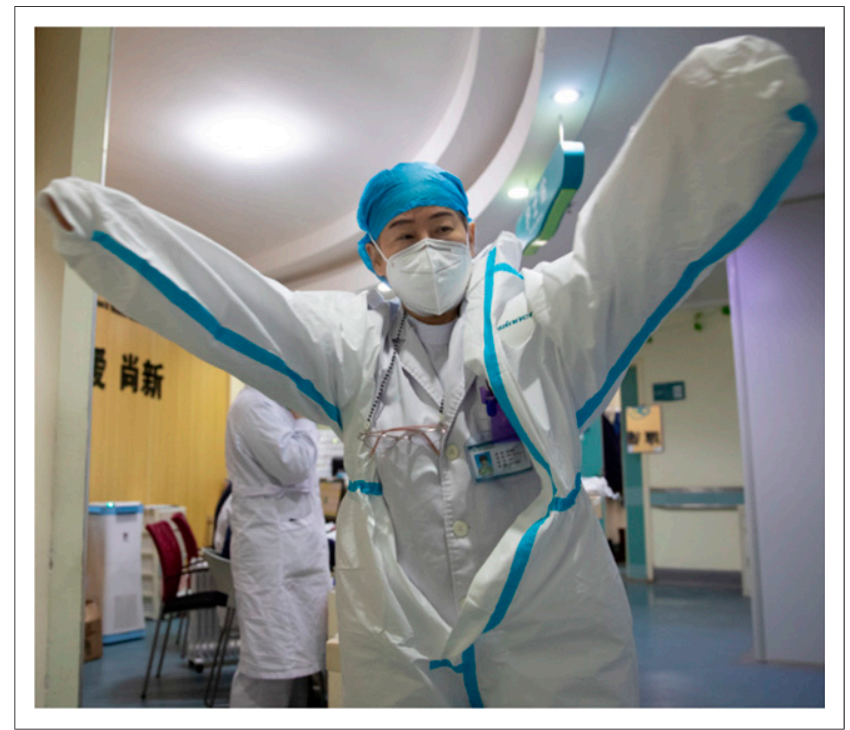

FIGURE 1. Zhang Jixian, director of the respiratory and critical care medicine department of Hubei Provincial Hospital of Integrated Chinese and Western Medicine, puts on the protective suit before entering the ward at the hospital in Wuhan, central China's Hubei Province, March 11, 2020. 